\title{
Aspects of the Cytology, Chemical Composition and Elemental Distribution in Leaves of Ni-Hyperaccumulating and Non-Hyperaccumulating Genotypes of Senecio coronatus
}

\author{
J. Mesjasz-Przybylowicz ${ }^{1}$, A.D. Barnabas ${ }^{1}$, I. Yousef ${ }^{2}$, P. Dumas ${ }^{2}$, F. Jamme ${ }^{2}$, T.P. Sechogela ${ }^{1}$, \\ W.J. Przybylowicz ${ }^{1,3}$ \\ 1. Materials Research Dept., iThemba LABS, PO Box 722, 7129 Somerset West, South Africa \\ 2. Soleil Synchrotron, BP 48 F91192 Gif sur Yvette Cedex, France \\ 3. On leave from AGH University of Science and Technology, Faculty of Physics \& Applied \\ Computer Science, al. A. Mickiewicza 30, 30-059 Krakow, Poland
}

Hyperaccumulation is an unusual plant response to soils enriched with heavy metals. Such soils like those derived from ultramafic rocks, are characterized by elevated concentrations of heavy metals, mainly $\mathrm{Ni}, \mathrm{Co}, \mathrm{Zn}$ and $\mathrm{Cd}$. Most of the plants growing on these metal-rich soils exclude metals from their shoots as excessive accumulation of heavy metals is toxic to the majority of plants. However, about $2 \%$ of plants growing on metaliferous soils take up and accumulate large quantities of metals in their shoots: a phenomenon known as hyperaccumulation. Senecio coronatus, a widespread South African plant, also occurs on ultramafic outcrops where two genotypes have been identified: one hyperaccumulating Ni and the other lacking this ability. The aim of the present study was to compare aspects of the cytology, chemical composition and elemental distribution in these genotypes and to ascertain whether any differences could be related to their differential uptake of $\mathrm{Ni}$.

Cell cytology was investigated at the light microscope level using hand-cut sections of fresh leaves as well as sections of resin-embedded leaves. Chemical composition of some cellular components was ascertained using standard histochemical tests. Additional chemical composition information of the leaves was obtained by synchrotron infrared microspectroscopy on thin vibratomed leaf sections at the SOLEIL Synchrotron Facility in France (SMIS beam line). Concentration of $\mathrm{Ni}$ and its distribution in leaf tissues were determined with a nuclear microprobe (Materials Research Department, iThemba LABS, South Africa) on cryofixed and freeze-dried leaf sections. Two complementary techniques, particle induced X-ray emission (PIXE) and proton backscattering (BS) were performed simultaneously.

Cytologically, the main differences between leaves of the genotypes related to spherical bodies were present within vacuoles of mesophyll cells. In the Ni-hyperaccumulator, these were large and occurred most frequently in palisade mesophyll cells closest to the upper epidermis (Fig.1) whilst in the non-hyperaccumulator they were smaller and more sparsely distributed (Fig.2). Spherical bodies stained positively for lipids. Spectra acquired from infrared microspectroscopy did not indicate significant differences in major macromolecular classes such as proteins and carbohydrates in leaf tissues of the genotypes. However, because of the histochemical identification of lipids in mesophyll cells, particular attention was paid to the infrared absorbance band representing ester carbonyl stretching (spectral region $1770-1700 \mathrm{~cm}^{-1}$ ) attributed mainly to lipids and lignin [1]. From representative infrared spectra corresponding to the pixels at the cross-hairs (Figs. 3,6, arrows) of the visible images, chemical maps depicting the distribution and 
relative concentration of these functional groups in each genotype (Figs.4,7), were constructed. The strongest ester carbonyl absorbance occurred in the hyperaccumulator (Fig.4). An overlay of the chemical image over the visible image (Fig. 5) shows that the strong absorbance areas coincide with mesophyll cells, particular those closest to the upper epidermis and the vascular bundle regions. In the non-hyperaccumulator (Fig.7) there were fewer strong ester carbonyl absorbance areas and these coincided with the same tissue regions as seen in the hyperaccumulator (Fig.8). On the basis of the cytological data presented, it is likely that many of the strong ester carbonyl absorbance areas represent the presence of lipids (spherical bodies) and lignin (vascular bundles). Nuclear microprobe studies showed that the highest Ni enrichment was in the epidermis of the hyperaccumulator with lower $\mathrm{Ni}$ concentrations in other leaf tissues (Fig.9). Significantly lower concentrations of Ni were present in the leaf epidermis and most other leaf tissues of the non-hyperaccumulator (Fig.10). It is not clear at present if the differences in cytology and chemical composition of the genotypes are related to their differential uptake of Ni. Further research to clarify this is being undertaken.

\section{References}

[1] W.H.Nelson, Modern Techniques for Rapid Biological Analysis, VCH Publishers ,NY,1991.
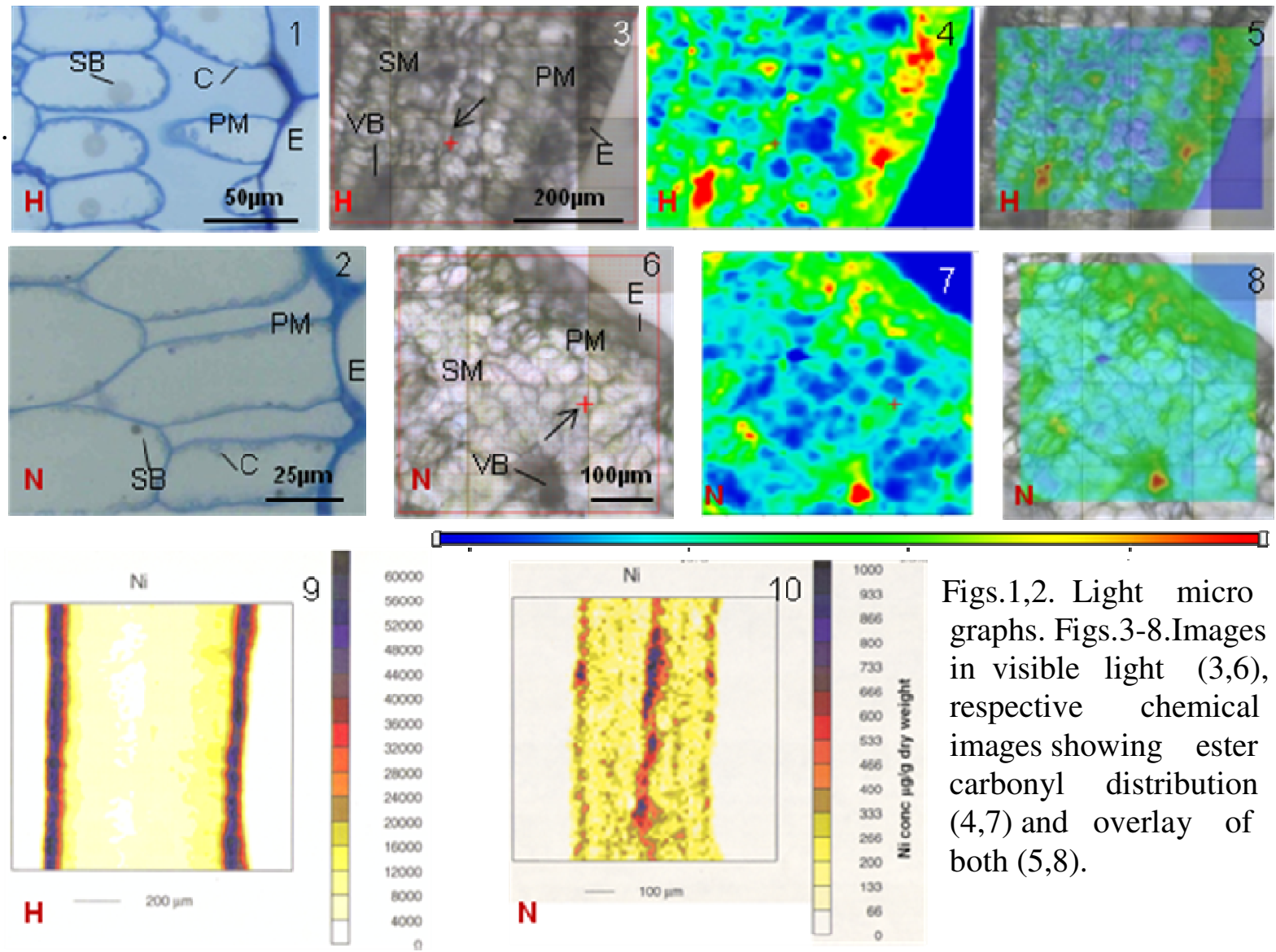

Figs.1,2. Light micro graphs. Figs.3-8.Images in visible light $(3,6)$, respective chemical images showing ester carbonyl distribution $(4,7)$ and overlay of both $(5,8)$.

Figs.9,10. Quantitative elemental maps of Ni distribution. The warmer colours in Figs.4,5,7,8 (towards the red end of the colour scale) indicate higher absorbance. C, chloroplasts; E, epidermal cells; $\mathrm{H}$, nickel hyperaccumulator; $\mathrm{N}$, non-hyperaccumulator; PM, palisade mesophyll; SB, spherical bodies; SM, spongy mesophyll; VB, vascular bundles. 